\title{
Outcomes of Transarterial Chemoembolization With or Without Additional Radiofrequency Ablation in Hepatocellular Carcinoma of 2
} to $5 \mathrm{~cm}$ in Diameter

\author{
Mi-Young Kim, ${ }_{1}$ Jin Woong Kim, ${ }^{2}$ Dae-Seong Myung, ${ }_{1}^{1}$ Chung-Hwan Jun, ${ }^{1}$ Wan-Sik Lee, ${ }^{1}$ Yang Jun Kang, ${ }^{2}$ \\ Sung-Kyu Choi, ${ }^{1}$ Young-Eun Joo, ${ }^{1}$ and Sung-Bum Cho, ${ }^{1,}$ \\ ${ }^{1}$ Departments of Internal Medicine, Chonnam National University Medical School, Gwangju, Korea \\ ${ }^{2}$ Departments of Radiology, Chonnam National University Medical School, Gwangju, Korea \\ "Corresponding author: Sung-Bum Cho, Departments of Internal Medicine, Chonnam National University Medical School, Gwangju, Korea. Tel: +82-622206296, Fax: \\ +82-622258578, E-mail: portalvein@naver.com
}

Received 2016 June 11; Revised 2016 August 04; Accepted 2016 August 15.

\begin{abstract}
Background: Hepatocellular carcinoma (HCC) is worldwide one of the most common and lethal malignant tumors despite attempts at treatment using various therapeutic modalities. Combination of transarterial chemoembolization (TACE) and radiofrequency ablation (RFA) can have expanded indication as curative therapy in patients with larger size $\mathrm{HCC}(2-5 \mathrm{~cm})$ that is unsuitable for RFA alone, but there are few studies showing long-term survival and larger sample size.

Objectives: The goal of this study was to evaluate the long-term efficacy and safety of combined TACE with RFA, and TACE alone in hepatocellular carcinoma of 2 to $5 \mathrm{~cm}$.

Patients and Methods: This was a retrospective study including 207 consecutive patients who were enrolled using computerized hepatocellular carcinoma database consisting of 105 patients who underwent combined TACE with RFA, and 102 patients who underwent TACE alone with long-term follow-up.

Results: The complete remission rate was meaningfully higher in the combination group $(97.1 \%, 102 / 105)$ than in the TACE group $(54.9 \%, 56 / 102)(\mathrm{P}<0.001)$. The mean follow-up periods of the combination group and the TACE group were $49.3 \pm 16.9$ months and $46.3 \pm 26.7$ months, respectively. The TACE group (90.2\%, 92/102) showed significantly higher tumoral recurrence or persistence than the combination group $(59.0 \%, 62 / 105)$ during follow-up periods $(\mathrm{P}<0.001)$. The cumulative survival rates at 1, 2, 3, 4, and 5 years were 88.6, 82.9, 79.0, 75.2, and 74.3\%, respectively in the combination group and 93.1, 73.5, 59.8, 50.0, and 45.1\%, respectively in the TACE group. Independent factors associated with improved overall survival were the combination group, Child-Pugh class A, complete remission at 1 month, negative intrahepatic new tumors, and no adverse event.

Conclusion: Complete local tumor control by combination of TACE with RFA could improve overall survival in comparison with TACE alone for long-term follow-up. The combination of TACE with RFA should be considered for achieving complete local tumor control before progression to advanced stage in HCC of 2 to $5 \mathrm{~cm}$.
\end{abstract}

Keywords: Hepatocellular Carcinoma, Radiofrequency Ablation, Transarterial Chemoembolization

\section{Background}

Hepatocellular carcinoma (HCC) is known worldwide as one of the most common and fatal malignant tumors despite attempts at treatment using various therapeutic modalities (1). The diagnosis of early HCC is increasing in endemic countries because of regular surveillance using multidetector computed tomography (CT) or magnetic resonance imaging (MRI) in high-risk groups (2). The choice of treatment is determined with many clinical considerations according to liver function, tumor status like size, number and vascular invasion, and patient status, such as performance score. Surgical resection or liver transplantation (LT) is providing opportunities for com- plete cure of early hepatocellulcar carcinoma, but because of the high recurrence rate after surgery and invasiveness of the modality, it has caused reluctance.

Radiofrequency ablation (RFA) is also safe and effective for the treatment of small HCC less than $2 \mathrm{~cm}$ and is preferred as a minimal invasive treatment. However, the role of RFA is limited in patients with a tumor larger than $2 \mathrm{~cm}$ because the rate of local progression is significantly increased depending on the size of the HCC (3). Combination of transarterial chemoembolization (TACE) and RFA has been successful to a local cancer control in early hepatocellular carcinoma, particularly for patients with HCC $2-5 \mathrm{~cm}$ in size (4). Combination of TACE and RFA can have expanded indication as curative therapy in 
patients with larger size HCC $(2-5 \mathrm{~cm})$ that is unsuitable for RFA alone. In many clinical studies, TACE combined with RFA therapy also demonstrated improved overall survival and local control rates in comparison to TACE alone in patients with larger HCC or meeting Milan criteria (59). However, because there are few studies showing longterm survival and larger sample size, most guidelines including Barcelona clinic liver cancer (BCLC) stage recommend TACE monotherapy for patients with HCC over $3 \mathrm{~cm}$ regarded as intermediate stage despite the advantage of combination treatment for the prognosis of patients.

\section{Objectives}

The purpose of the current study was to assess longterm efficacy and safety of TACE combined with RFA and TACE alone in patients with single or two HCCs of 2 to $5 \mathrm{~cm}$.

\section{Patients and Methods}

\subsection{Patient Population}

A constructed computerized HCC database was used to identify 3,125 patients who had visited our hospital between January 2007 and December 2011. Informed consent was obtained from each patient before treatment. Diagnosis of HCC was based on $\alpha$-fetoprotein (AFP) levels, standard clinical and imaging criteria according to the practice guidelines of the European association for the study of the liver (EASL) (10). The treatment modalities were separately decided by three hepatologists with discussion to an interventional radiologist in patients with $\mathrm{HCC} 2-5 \mathrm{~cm}$ in diameter who would be unsuitable for surgical resection. This was a historical cohort study consisting of 207 consecutive patients who met the following criteria. Of these, 105 patients who underwent combined TACE with RFA and 102 patients who underwent TACE alone were included in the study (Figure 1). The inclusion criteria were as follows: i, Number of tumors was single or two; ii, Size of tumors ranged between $2 \mathrm{~cm}$ and $5 \mathrm{~cm}$ in the largest dimension; iii, Child-Pugh class A or B; iv, Duration of follow up was more than 36 months; $v$, eastern cooperative oncology group performance status (ECOG) 0 or 1. Exclusion criteria were as follows: i, Other treatment modalities including initial surgical treatment or RFA only; ii, ChildPugh class C; iii, Radiologic evidence of vascular invasion and metastasis; vi, Severe extrahepatic comorbidities. The study was approved by the institutional review board.

\subsection{Transarterial Chemoembolization (TACE Group)}

Interventional radiologist initially performed superior mesenteric and common hepatic arteriographies to assess tumor burden, patient anatomy, and vascularity. After identifying the feeding arteries, chemoembolization of the feeding arteries was carried out through superselective catheterization as near to the hypervascular mass as possible. A mixture of doxorubicin hydrochloride (Adriamycin;Ildong Co. Ltd., Seoul, Korea) and an emulsion of iodized oil (Lipiodol; Laboratoire Guerbet, Aulnay Sous Bois, France) was used in the chemoembolization. The amount of iodized oil was determined according to the size of the HCC (1 cc per $1 \mathrm{~cm}$ diameter of the tumor) and the maximum capacity of the iodized oil was $10 \mathrm{cc}$. The infusion of the mixture was carried out up to the stagnation of arterial flow, followed by infusion of Gelfoam powder (Cutanplast; Mascia Brunelli,Milan, Italy).

\subsection{Radiofrequency Ablation After TACE (Combined TACE With RFA Group)}

RFA was performed under ultra-sonographic guidance the next day after TACE. All patients received RFA procedure under sedation and local anesthesia. A monopolar radiofrequency generator (CC-1, Valleylab,Boulder, CO, USA) and a $17 \mathrm{G}$ dual clustered internally cooled electrode with a $3 \mathrm{~cm}$ exposed tip (STARmed Co. Ltd.; Goyang, Korea) were used for RFA. If the repeated insertion of electrode was required because of the geometry of the tumor, $17 \mathrm{G}$ dual clustered, $17 \mathrm{G}$ single and/or $15 \mathrm{G}$ single internally cooled electrode with $3 \mathrm{~cm}$ exposed tip (STARmed Co. Ltd.; Goyang, Korea) were additionally used. In cases where the tumor was located close to the diaphragm or the colon, artificial ascites using normal saline was routinely implanted in the process of inserting electrodes. Radiofrequency current was emitted through the generator set to provide the maximal power in the impedance control mode automatically. Tumor ablation lasted for 8 to 12 minutes at each electrode placement. RFA was terminated when the complete ablation of the visible tumor was achieved on ultrasound images. The electrode track was ablated to prevent bleeding and tumor seeding at the end of the procedure (Figure 2).

Within 3 hours of RFA, contrast-enhanced CT was performed. If tumoral enhancements near the ablation zone were found, RFA was performed additionally for the residual tumor.

\subsection{Follow-Up}

Dynamic enhanced CT was performed within 1 month for evaluation of therapeutic response defined by the absence of an enhanced tumor area. All patients received CT every three months for two years and every six months 


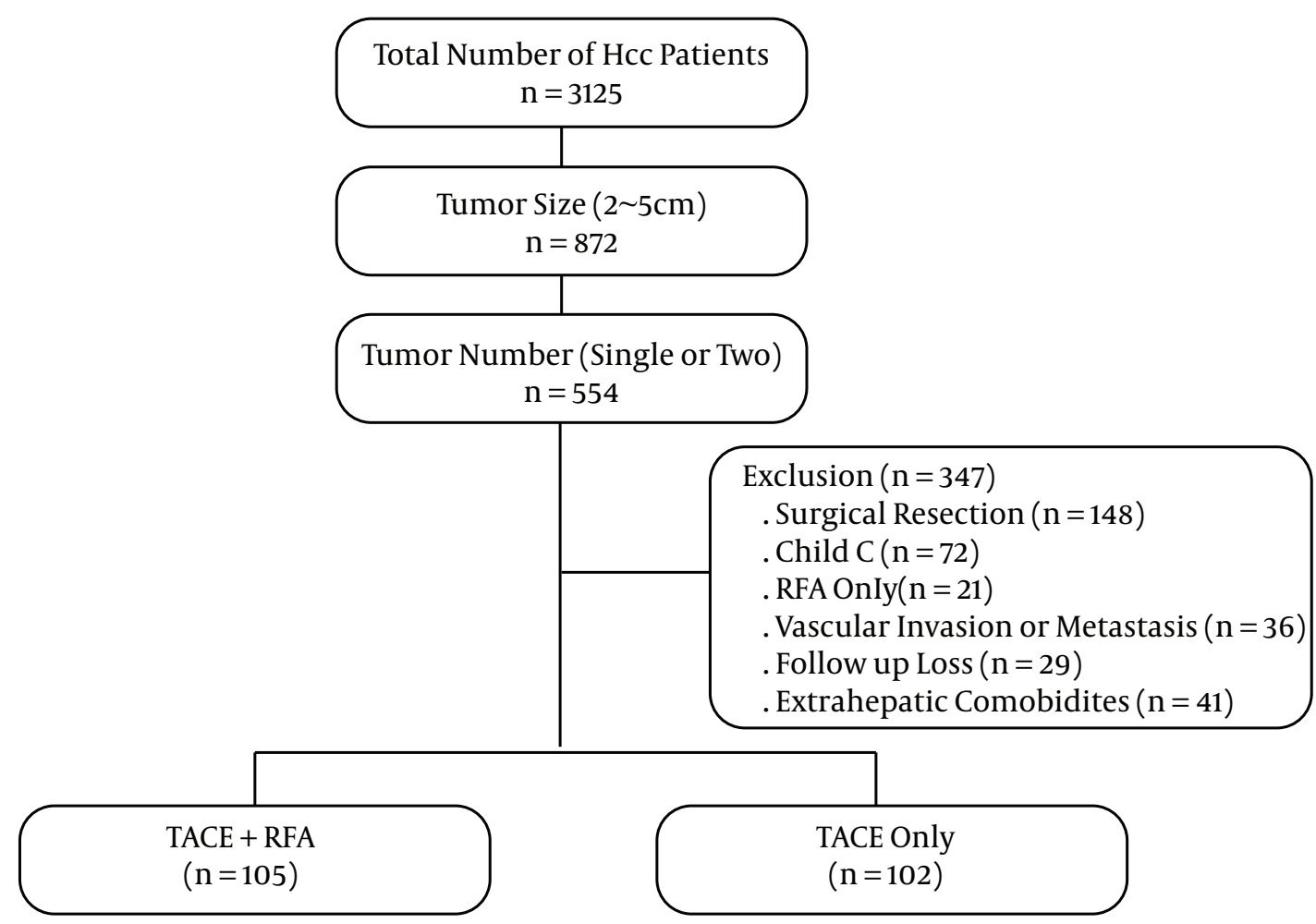

Figure 1. Flow chart of enrolled hepatocellular carcinoma (HCC) patients who underwent combined transarterial chemoembolization (TACE) with radiofrequency ablation (RFA), and TACE alone according to tumor size and number.

after two years (Figure 3). Blood tests including a serum alpha-fetoprotein (AFP), liver function tests, and physical examination were conducted at each visit. If the lesion showing early enhancement and late washout was found in another area of the liver on CT scan, it was diagnosed as a newly developed HCC. When there was a new tumor located in contact with the original tumor, it was defined as local recurrence of the tumor.

\subsection{Statistical Analysis}

SPSS 18.0 software (SPSS, Chicago, IL) was used for statistical analysis. The groups were compared using the $\chi^{2}$ test or Fisher's exact test for categorical data, and student's t test or the Mann-Whitney $U$-test for continuous data. The Kaplan-Meier method and the log rank test were used for calculation and comparison of overall survival and recurrence free survival. Statistical significance was accepted for P values $<0.05$.

\section{Results}

\subsection{Baseline Characteristics of the Patients}

Table 1 shows the baseline characteristics of the patients. There were no significant differences in terms of age, sex, etiology, previous treatment, mean tumor size, tumor number, and alpha-fetoprotein (AFP) between the two groups. The significant clinical factors affecting decision of two treatment modalities were the underlying liver function and tumor location. The TACE group included more patients with poor liver function (child $B, n=20$ ) than the combination group (child $B, n=7$ ) and more frequent tumor locations in the S1, S3, and S5 segments of liver related to RFA difficulty as approach of RFA tip, vascular abutting, and surface location (Table 1).

\subsection{Clinical Outcomes}

The complete remission rates were significantly higher in the combination group $(97.1 \%, 102 / 105)$ than in the TACE group $(54.9 \%, 56 / 102)$ by dynamic enhanced CT within 1 month of evaluation of tumor response $(\mathrm{P}<0.001)$. The mean follow-up periods of the combination group and 

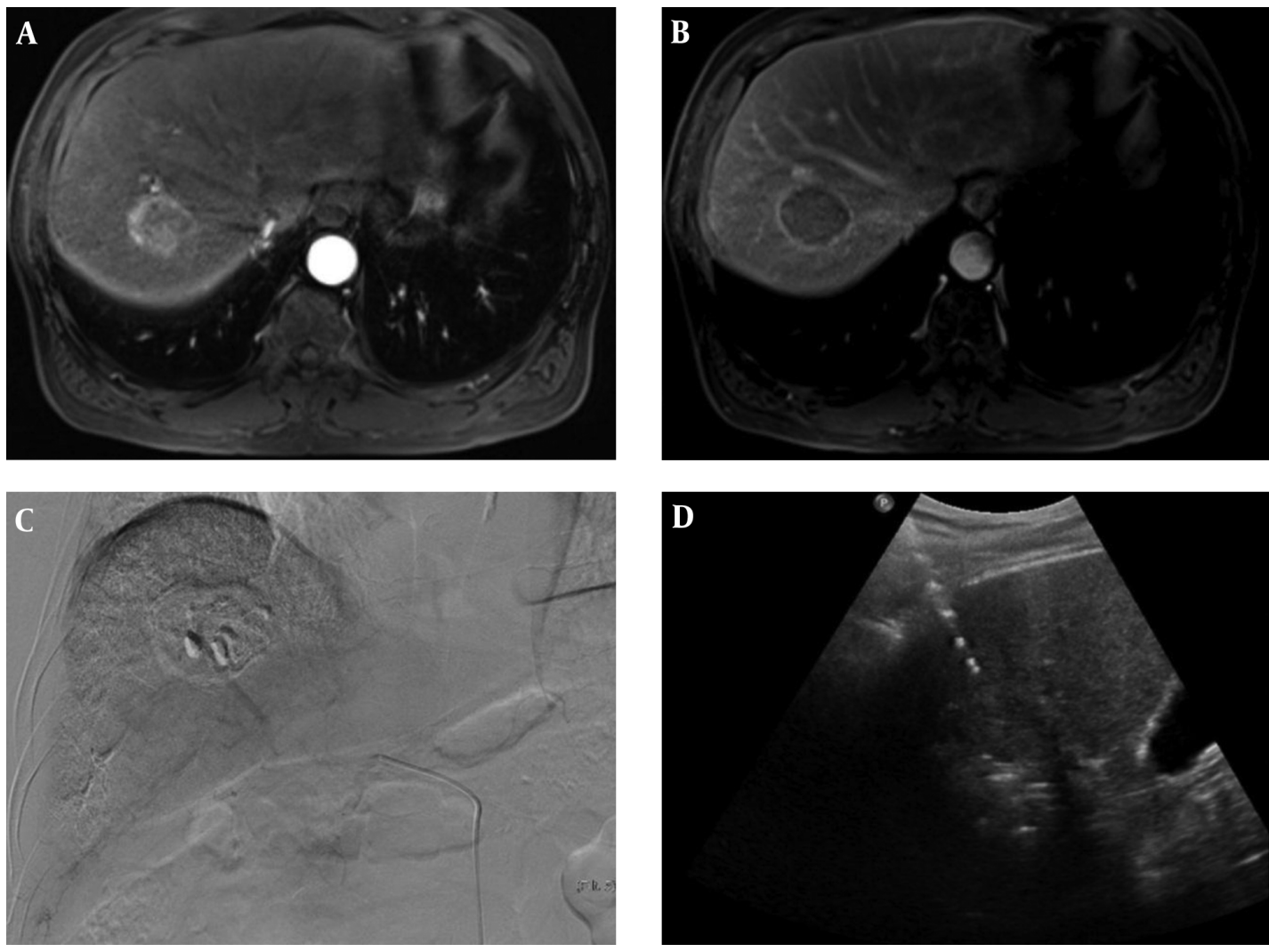

Figure 2. A 70-year-old man with $4 \mathrm{~cm}$ hepatocellular carcinoma (HCC), who underwent combined transarterial chemoembolization (TACE) and radiofrequency ablation (RFA). A, B. MR images shows a $4 \mathrm{~cm}$ sized, well-defined mass in liver segment 7. The mass was diagnosed with hepatocellular carcinoma due to enhancement on the arterial phase (A) and washout on the delayed phase with an enhancing capsule (B). C. Post-TACE angiogram shows dense radiopaque mass with accumulated iodized oil in the right hepatic lobe. D. Ultrasonography image shows mass and echogenic radiofrequency electrode within mass during RFA.
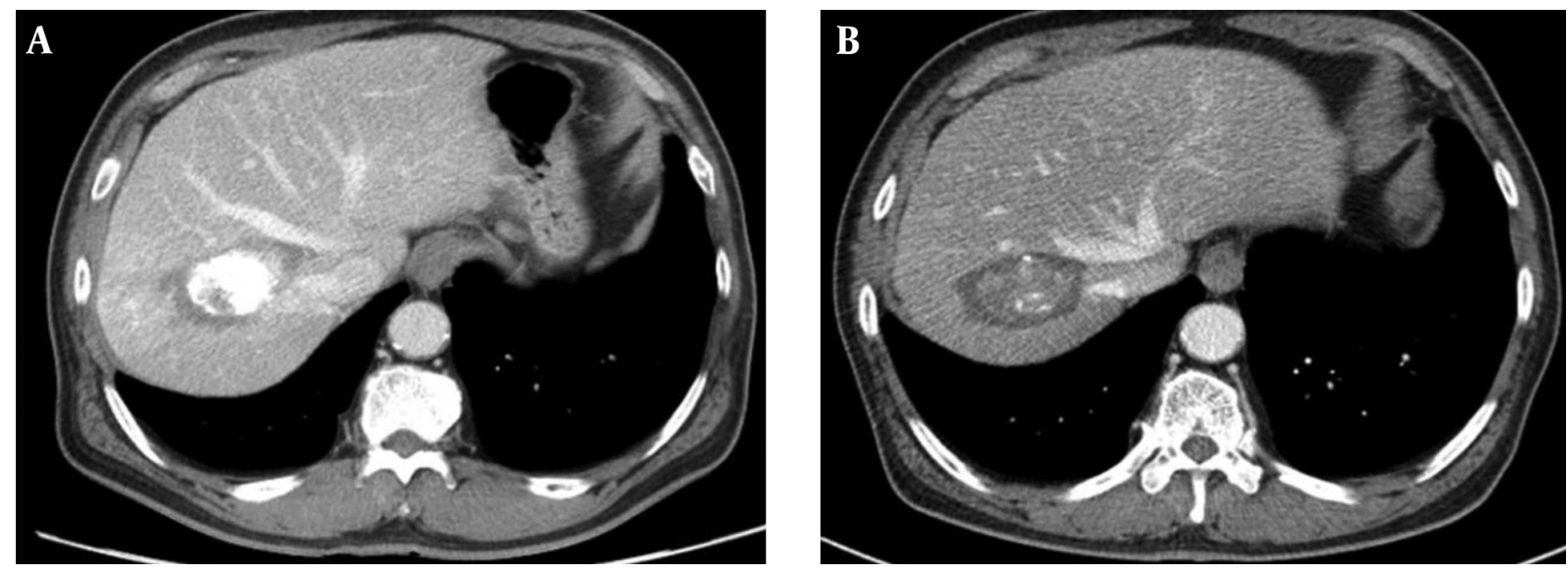

Figure 3. Contrast-enhanced CT images obtained 1 month (A), and 5 years (B) after combined therapy show dense iodized oil accumulation in the mass surrounded by radiofrequency induced coagulation without local tumor progression.

TACE group were $49.3 \pm 16.9$ months and $46.3 \pm 26.7$ months, respectively. The mean numbers of additional RFA or TACE after initial treatment were more frequent in the TACE group ( $5.1 \pm 3.3)$ than in the combination group (2.3 
Table 1. Baseline Features of the Patients Between Two Groups ${ }^{\mathrm{a}}$

\begin{tabular}{|c|c|c|c|}
\hline Variables & TACE + RFA, $(\mathbf{n}=\mathbf{1 0 5})$ & TACE, $(\mathbf{n}=102)$ & P-Value \\
\hline Age, $y,{ }^{b}$ & $63.4 \pm 9.7$ & $62.4 \pm 10.2$ & 0.605 \\
\hline Sex & & & 0.817 \\
\hline Male & 82 & 81 & \\
\hline Female & 23 & 21 & \\
\hline Etiology, (HBV/HCV/alcoholics/other) & $71 / 17 / 11 / 6$ & $60 / 20 / 19 / 3$ & 0.181 \\
\hline Primary/recurrent & $95 / 10$ & $88 / 14$ & 0.345 \\
\hline Positive HBV DNA & 41 & 44 & 0.510 \\
\hline Antiviral drug for $\mathrm{HBV}$ & 36 & 29 & 0.541 \\
\hline Persist alcohol abuse & $8 / 37$ & $9 / 41$ & 0.936 \\
\hline Tumor diameter, mm, ${ }^{\text {b }}$ & $28.3 \pm 7.6$ & $28.7 \pm 9.2$ & 0.734 \\
\hline Number of tumors $(\mathbf{1} / 2)$ & $77 / 28$ & $80 / 22$ & 0.392 \\
\hline Tumor location, (segment 1/2/3/4/5/6/7/8) & $0 / 10 / 2 / 9 / 10 /, 19 / 23 / 32$ & $3 / 11 / 7 / 9 / 24 /, 7 / 17 / 24$ & 0.008 \\
\hline Child-Pugh class $(\mathrm{A} / \mathrm{B})$ & $98 / 7$ & $82 / 20$ & 0.006 \\
\hline AFP, ng/mL, ${ }^{\text {b }}$ & $905 \pm 3164$ & $697 \pm 2438$ & 0.617 \\
\hline ALT, IU/L, ${ }^{b}$ & $31.2 \pm 21.5$ & $34.1 \pm 23.7$ & 0.356 \\
\hline Total bilirubin, mg/L, & $0.68 \pm 0.41$ & $1.04 \pm 0.55$ & $<0.001$ \\
\hline Albumin, g/dL, ${ }^{\text {b }}$ & $4.19 \pm 0.44$ & $3.72 \pm 0.53$ & $<0.001$ \\
\hline PT (INR), ${ }^{\mathbf{b}}$ & $1.09 \pm 0.13$ & $1.18 \pm 0.15$ & $<0.001$ \\
\hline Platelet, $\times 10^{3} \mid \mu \mathbf{L},{ }^{\mathbf{b}}$ & $137.2 \pm 59.1$ & $110.9 \pm 51.9$ & 0.001 \\
\hline
\end{tabular}

$\pm 1.7)$ during follow-up periods $(\mathrm{P}<0.001)$. The TACE group (90.2\%, 92/102) showed significantly higher tumoral recurrence or persistence than the combination group $(59.0 \%$, $62 / 105)$ during follow-up periods $(\mathrm{P}<0.001)$. The original target tumor progression when detecting first time after treatment was meaningfully higher in the TACE group $(70.7 \%, 65 / 92)$ than in the combination group (35.5\%, 22/62). Twenty-seven patients (26.5\%) in the combination group and 67 patients (65.7\%) in the TACE group had deceased at the end of the follow-up $(\mathrm{P}<0.001)$ (Table 2$)$.

\subsection{Overall Survival and Recurrence Free Survival}

The cumulative survival rates at 1, 2, 3, 4, and 5 years were $88.6,82.9,79.0,75.2$, and $74.3 \%$, respectively in the combination group and 93.1, 73.5, 59.8, 50.0, and 45.1\%, respectively in the TACE group. A significantly better overall survival rate was observed for the combination group compared with the TACE group $(P=0.002)$ (Figure 4$)$. The rates of recurrence-free survival at 1, 2,3, 4, and 5 years were $61.9,54.3,47.6,42.9$, and $41.0 \%$, respectively in the combination group and $28.4,18.6,12.7,10.8$, and $9.8 \%$, respectively in the TACE group. The significantly better recurrence-free survival rate was also observed for the combination group compared with the TACE group $(\mathrm{P}<0.001)$ (Figure 5).

\subsection{Clinical Factors Associated with Overall Survival}

In univariate analysis, therapy group, Child-Pugh class, tumor size, treatment response at 1 month, and new intrahepatic tumors were significantly associated with overall survival. In multivariate analysis, independent factors significantly associated with better overall survival were combination group, Child-Pugh class A, complete remission at 1 month, negative new intrahepatic tumors, and no adverse event (Table 3).

\subsection{Complications}

The combination group had more frequent occurrence of procedure related adverse events than the TACE group. The procedure related severe adverse events were abscess (1.9\%, 2/105), hemoperitoneum $(1.9 \%, 2 / 105)$, hepatic failure $(1.0 \%, 1 / 105)$, delayed colon perforation (1.0\%, 1/105), pneumothorax (1.0\%, 1/105), and segmental hepatic infarction 
Table 2. Comparison of Clinical Outcomes Between Two Groups ${ }^{\mathrm{a}}$

\begin{tabular}{|c|c|c|c|}
\hline Variables & TACE + RFA, $n=105(\%)$ & TACE, $\mathbf{n}=\mathbf{1 0 2}(\%)$ & P Value \\
\hline Complete remission in 1 month & $102(97.1)$ & $56(54.9)$ & $<0.001$ \\
\hline Mean duration of follow up (month), & $49.3 \pm 16.9$ & $46.3 \pm 26.7$ & $<0.001$ \\
\hline Mean number of additional TACE or RFA, ${ }^{\text {b }}$ & $2.3 \pm 1.7$ & $5.1 \pm 3.3$ & $<0.001$ \\
\hline Total recurrence & $62(59.0)$ & $92(90.2)$ & $<0.001$ \\
\hline $1-2$ years & $8(12.9)$ & $10(10.9)$ & \\
\hline $2-3$ years & $7(11.3)$ & $6(6.5)$ & \\
\hline $3-4$ years & $5(8.1)$ & $2(2.2)$ & \\
\hline $4-5$ years & $2(3.2)$ & $1(1.1)$ & \\
\hline The characteristics of recurrence at first timing & & & $<0.001$ \\
\hline Target tumor progression & $22(35.5)$ & $65(70.7)$ & \\
\hline Intrahepatic new lesion & $36(58.1)$ & $20(21.7)$ & \\
\hline Both & $4(6.4)$ & $7(7.6)$ & \\
\hline Total death & $27(26.5)$ & $67(65.7)$ & $<0.001$ \\
\hline Death of non-hepatic cause & $1(3.7)$ & $4(6.0)$ & 0.816 \\
\hline
\end{tabular}

Abbreviations: RFA, radiofrequency ablation; TACE, transarterial chemoembolization.

${ }^{\mathrm{a}}$ Values are expressed as No. (\%).

${ }^{\mathrm{b}}$ Data are expressed as means \pm standard deviations.

Table 3. Clinical Factors Associated with Overall Survival in Univariate and Multivariate Analyses

\begin{tabular}{|c|c|c|c|c|c|c|}
\hline & \multicolumn{3}{|c|}{ Univariate Analysis } & \multicolumn{3}{|c|}{ Multivariate Analysis } \\
\hline & HR & $(95 \% \mathrm{CI})$ & P-Value & HR & $(95 \% \mathrm{CI})$ & P-Value \\
\hline Age & 0.985 & $(0.967-1.004)$ & 0.985 & & & \\
\hline Sex & 1.005 & $(0.607-1.666)$ & 0.984 & & & \\
\hline Therapy, (TACE only Vs. TACE+RFA) & 2.006 & $(1.273-3.160)$ & 0.003 & 1.922 & $(1.163-3.177)$ & 0.011 \\
\hline Tumor number, (1: 2) & 1.252 & $(0.792-1.979)$ & 0.336 & & & \\
\hline Tumor size, $(\mathrm{mm} ; \leq 30:>30)$ & 1.684 & $(1.122-2.526)$ & 0.012 & 1.513 & $(0.993-2.306)$ & 0.054 \\
\hline Tumor location & 1.033 & $(0.938-1.138)$ & 0.504 & Variables & & \\
\hline Complete remission at 1 month & 2.204 & $(1.454-3.340)$ & $<0.001$ & 1.782 & $(1.103-2.881)$ & 0.018 \\
\hline Local tumor progression & 1.068 & $(0.406-2.808)$ & 0.895 & & & \\
\hline Severe adverse events & 2.257 & $(0.819-6.215)$ & 0.115 & 4.032 & $(1.366-11.903)$ & 0.012 \\
\hline
\end{tabular}

Abbreviations: CI, confidence interval; RFA, radiofrequency ablation; TACE, transarterial chemoembolization

$(1.0 \%, 1 / 105)$ in the combination group alone. Three patients who had developed abscess, hepatic failure, and colon perforation died within 3 months after combination treatment $(2.9 \%, 3 / 105)$. Elevation of aminotransferase and fever in both groups were common adverse events. However, the combination group had a significantly higher eleva- tion of aminotransferase over 5 times (81.9\%, 86/105) and high fever $(29.5 \%, 31 / 105)$ compared with the TACE group (11.8\%. 12/105 and 11.8\%, 12/102) (Table 4). 
Table 4. Adverse Events of Two Groups ${ }^{\mathrm{a}}$

\begin{tabular}{|c|c|c|c|}
\hline Variables & TACE + RFA Group, $\mathbf{n}=105(\%)$ & TACE Group, n = 102 (\%) & P-Value \\
\hline Duration of admission (day), mean \pm SD & $8.3 \pm 3.6$ & $5.8 \pm 3.2$ & $<0.001$ \\
\hline Procedure related mortality & $3(2.9)$ & $0(0)$ & 0.171 \\
\hline Severe adverse event & $8(7.6)$ & $0(0)$ & 0.004 \\
\hline Abscess & $2(1.9)$ & $0(0)$ & \\
\hline Hemoperitoneum & $2(1.9)$ & $0(0)$ & \\
\hline Hepatic failure & $1(1.0)$ & & \\
\hline Delayed colon perforation & $1(1.0)$ & $0(0)$ & \\
\hline Pneumothorax & $1(1.0)$ & $0(0)$ & \\
\hline Segmental hepatic infarction & $1(1.0)$ & $0(0)$ & \\
\hline Elevation of aminotransferase & & & $<0.001$ \\
\hline the baseline level $<2$ times & $5(4.8)$ & $60(58.8)$ & \\
\hline 2 - 3 times the baseline level & $6(5.7)$ & $21(20.6)$ & \\
\hline 3- 5 times the baseline level & $8(7.6)$ & $9(8.8)$ & \\
\hline$\geq 5$ times the baseline level & $86(81.9)$ & $12(11.8)$ & \\
\hline Fever & & & 0.002 \\
\hline None & $74(70.5)$ & $90(88.2)$ & \\
\hline Fever $\left(\geq 38^{\circ} \mathrm{C}\right)$ & $31(29.5)$ & $12(11.8)$ & \\
\hline Use of antibiotics & $19(18.1)$ & $9(8.8)$ & 0.051 \\
\hline
\end{tabular}

Abbreviations: RFA, radiofrequency ablation; SD, standard deviation; TACE, transarterial chemoembolization.

${ }^{\mathrm{a}}$ Values are expressed as No. (\%) unless otherwise indicated.

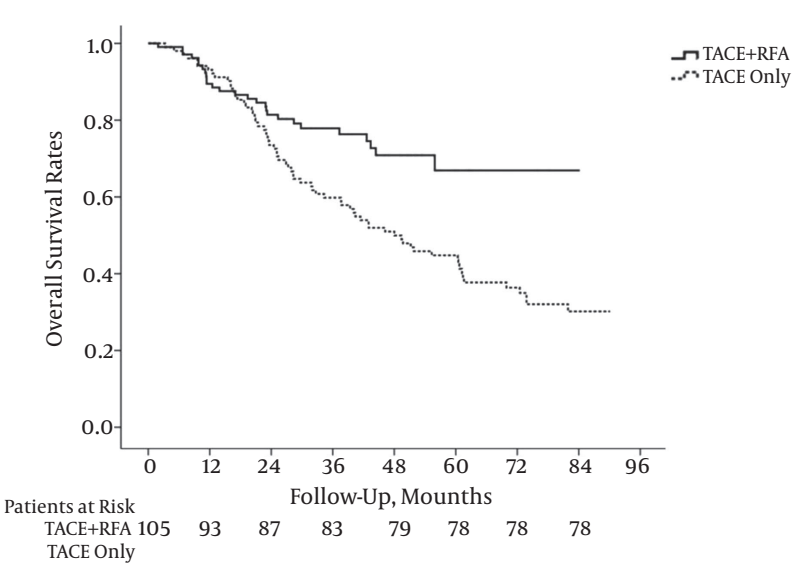

Figure 4. Kaplan-Meier curves show significant difference of overall survival rates between patients in two groups with early hepatocellular carcinoma ranging from 2 to $5 \mathrm{~cm}(P=0.002)$

\section{Discussion}

The initial tumor control that could affect overall clinical outcomes is very important in patients with early HCC before progressing to advanced stage. Many guidelines

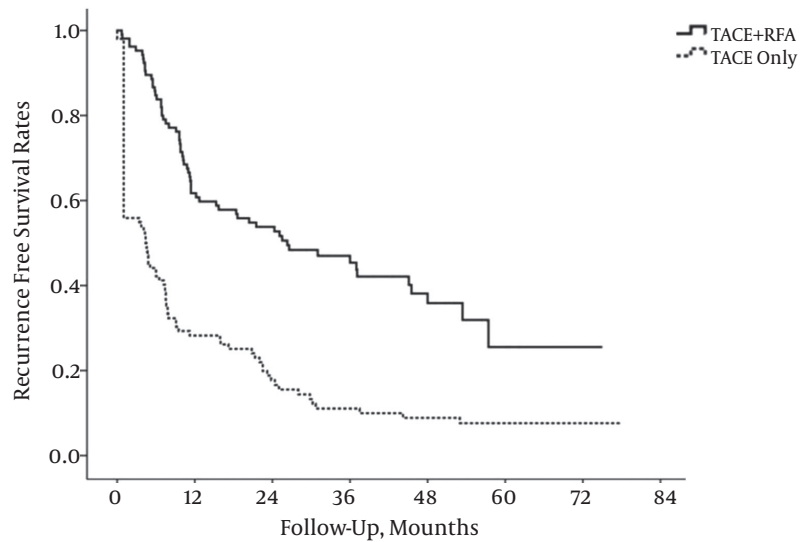

Figure 5. Kaplan-Meier curves show significant difference of recurrence free survival rates between patients in two groups with early hepatocellular carcinoma ranging from 2 to $5 \mathrm{~cm}(\mathrm{P}<0.001)$.

uniformly recommend non-curative TACE monotherapy for early HCC that would be unsuitable for surgery or RFA $(11,12)$. Surgical resection has remained discouraging under real clinical practices because of high postoperative re- 
currence rate and invasive modalities. TACE alone has been reported to be less effective than the combination of TACE with RFA in patients with early HCC (9, 13-15). However, there is still controversy for the optimal protocol and indication of combination therapy. And long term results are not fully established. The current study was conducted to assess the long term efficacy and safety of combined TACE with RFA and TACE alone in patients with single or two HCCs of 2 to $5 \mathrm{~cm}$ in size.

In case of small HCC of $3 \mathrm{~cm}$ or less, RFA alone achieves complete necrosis in more than $90 \%$. However, when the size of the tumor is more than $2 \mathrm{~cm}$, the possibility of local recurrence is higher due to the high incidence of microscopic viable tumor after RFA $(16,17)$. Combination of TACE with RFA has several advantages and its indication can be expanded to a larger size to provide better local tumor control than RFA alone. The decreased blood flow to an HCC induced by TACE may reduce the heat sink effect of tumor vasculature, resulting in an increased RFA ablation zone (18, 19). Moreover, the hypoxic injury and effect of chemotherapy induced by TACE increases vulnerability of tumor cells by high temperature during RFA (20), causing expansion of the ablated zone $(4,21)$. Combination of TACE with RFA therapy is more effective, particularly for HCCs $2-5 \mathrm{~cm}$ in size $(4,8,20)$. We previously reported that the effect of combined TACE with RFA is similar to that of surgical treatment in patients with a single HCC of 2 to $5 \mathrm{~cm}$ in size (4).

There has been no standardized protocol for the appropriate time interval between TACE and RFA. The time interval was various from the same day to two months in many studies $(4,16,20,22)$. Theoretically, when RFA is performed on the same day, the embolization effect of combination of TACE and RFA may be maximized. In this study, RFA was performed routinely 24 hours after TACE. We recommend that a one day interval between TACE and RFA could be helpful in maintaining balance regarding several concerns about risk of complications, additional advantages of hyperthermia on chemotherapy and decreasing the heat sink effect in tumor by embolization effect.

Complete local tumor control by initial therapy in patients with early HCC is very important in preventing progression to advanced stage concerning the balance of effects and complications. TACE alone has been used as a safe and effective treatment for early HCC unsuitable for curative modalities such as RFA alone or surgical resection. However, incomplete local tumor control by TACE alone for early HCCs eventually results in earlier progression to advanced stage and shows poor clinical outcomes in this study (9). The complete local tumor control rate was significantly higher in combined TACE (97.1\%) with RFA than in TACE alone (54.9\%), and significant differences in overall survival rates and recurrence-free survival were observed during long term follow-up. In this study, the overall survival rates at 1, 2, 3, 4, and 5 years were 88.6, 82.9, 79.0, 75.2, and $74.3 \%$, respectively in combined TACE with RFA and 93.1, $73.5,59.8,50.0$, and $45.1 \%$, respectively in TACE alone. The rates of recurrence-free survival at 1, 2, 3, 4, and 5 years were $61.9,54.3,47.6,42.9$, and $41.0 \%$, respectively in the combination group and $28.4,18.6,12.7,10.8$, and $9.8 \%$, respectively in the TACE group. Thus, we believe that combination of TACE and RFA should be considered as the first line treatment for patients with early hepatocellular carcinoma 2 - 5 $\mathrm{cm}$ in size and 1 - 2 in tumor number.

In terms of safety, the combination group (7.6\%) had more frequent occurrence of severe complications than the TACE group ( $0 \%$ ) because of expanding ablation zone. We experienced a few major complications including hepatic abscess, hemoperitoneum, and hepatic failure in the combination group. Three patients among the combination group died by procedure related complications within 3 months. Because the combination group had frequent elevation of aminotransferase more than 5 times (81.9\%) and complications, combination of TACE and RFA therapy should be carefully considered in HCC patients with Child-Pugh B liver function. This study showed more frequent major complications compared with other studies (0.4 - 5\%) in the combination group because of the short interval between TACE and RFA, larger size and number of tumors, and larger sample size (23-25).

This study has several limitations including retrospective study, selection bias, and discrepancy of BCLC guidelines. However, this study was focused on long term results and safety compared with TACE only through the expanding indication ( $2-5 \mathrm{~cm}$ size) of nonsurgical curative options. A large randomized multicenter study is required to verify the clinical implications and precise protocols of the combined modalities.

In conclusion, combination of TACE and RFA therapy should be considered for achieving complete local tumor control before progression to advanced stage in patients with HCC $2-5 \mathrm{~cm}$ in size. Complete local tumor control by combination of TACE with RFA therapy could improve overall survival compared with TACE alone for long-term follow-up. A large randomized multicenter study is required to verify the clinical implications and precise protocols of the combined modalities.

\section{Acknowledgments}

None. 


\section{Footnotes}

Authors' Contributions: Mi-Young Kim and Jin Woong Kim contributed equally in this study. Study concept and design: Mi-Young Kim, Jin-Woong Kim, Sung-Bum Jo, Young-Eun Joo, Sung-Kyu Choi, and Wan-Sik Lee; acquisition of data: Mi-Young Kim, Jin-Woong Kim, Dea-Seong Myung, and Chung-Hwan Jun; analysis and interpretation of data: Mi-Young Kim, Jin-Woong Kim, Sung-Bum Jo, Young-Eun Joo, Sung-Kyu Choi, and Wan-Sik Lee; drafting of the manuscript: Mi-Young Kim, Jin-Woong Kim, and SungBum Jo; critical revision of the manuscript for important intellectual content: Mi-Young Kim, Jin-Woong Kim, and Sung-Bum; statistical analysis: Sung-Bum Jo; administrative, technical, and material support: Jin-Woong Kim, YangJun Kang, and Sung-Bum Jo; study supervision:Sung-Bum Jo

\section{Funding/Support: None declared.}

Financial disclosure: None declared.

\section{References}

1. Forner A, Llovet JM, Bruix J. Hepatocellular carcinoma. Lancet. 2012;379(9822):1245-55. doi: 10.1016/S0140-6736(11)61347-0. [PubMed: 22353262].

2. Santi V, Trevisani F, Gramenzi A, Grignaschi A, Mirici-Cappa F, Del Poggio $P$, et al. Semiannual surveillance is superior to annual surveillance for the detection of early hepatocellular carcinoma and patient survival. J Hepatol. 2010;53(2):291-7. doi: 10.1016/j.jhep.2010.03.010. [PubMed: 20483497].

3. Takaki H, Yamakado K, Sakurai H, Nakatsuka A, Shiraki K, Isaji S, et al. Radiofrequency ablation combined with chemoembolization: treatment of recurrent hepatocellular carcinomas after hepatectomy. AJR Am JRoentgenol. 2011;197(2):488-94. doi:10.2214/AJR.10.4933. [PubMed: 21785099].

4. Kim JW, Shin SS, Kim JK, Choi SK, Heo SH, Lim HS, et al. Radiofrequency ablation combined with transcatheter arterial chemoembolization for the treatment of single hepatocellular carcinoma of 2 to $5 \mathrm{~cm}$ in diameter: comparison with surgical resection. Korean J Radiol. 2013;14(4):626-35. doi:10.3348/kjr.2013.14.4.626. [PubMed: 23901320].

5. Kirikoshi H, Saito S, Yoneda M, Fujita K, Mawatari H, Uchiyama T, et al. Outcome of transarterial chemoembolization monotherapy, and in combination with percutaneous ethanol injection, or radiofrequency ablation therapy for hepatocellular carcinoma. Hepatol Res. 2009;39(6):553-62. doi: 10.1111/j.1872-034X.2009.00490.x. [PubMed: 19527484].

6. Buscarini L, Buscarini E, Di Stasi M, Quaretti P, Zangrandi A. Percutaneous radiofrequency thermal ablation combined with transcatheter arterial embolization in the treatment of large hepatocellular carcinoma. Ultraschall Med. 1999;20(2):47-53. doi:10.1055/s-199914233. [PubMed: 10407974].

7. Peng ZW, Zhang YJ, Chen MS, Xu L, Liang HH, Lin XJ, et al. Radiofrequency ablation with or without transcatheter arterial chemoembolization in the treatment of hepatocellular carcinoma: a prospective randomized trial. J Clin Oncol. 2013;31(4):426-32. doi: 10.1200/JCO.2012.42.9936. [PubMed: 23269991].

8. Kim JH, Won HJ, Shin YM, Kim SH, Yoon HK, Sung KB, et al. Mediumsized (3.1-5.0 cm) hepatocellular carcinoma: transarterial chemoembolization plus radiofrequency ablation versus radiofrequency abla- tion alone. Ann Surg Oncol. 2011;18(6):1624-9. doi: 10.1245/s10434-0111673-8. [PubMed: 21445671].

9. Azuma S, Asahina Y, Nishimura-Sakurai Y, Kakinuma S, Kaneko S, Nagata $\mathrm{H}$, et al. Efficacy of additional radiofrequency ablation after transcatheter arterial chemoembolization for intermediate hepatocellular carcinoma. Hepatol Res. 2016;46(4):312-9. doi: 10.1111/hepr.12566. [PubMed: 26224167].

10. European Association For The Study Of The L, European Organisation For R, Treatment Of C. EASL-EORTC clinical practice guidelines: management of hepatocellular carcinoma. J Hepatol. 2012;56(4):908-43. doi: 10.1016/j.jhep.2011.12.001. [PubMed: 22424438].

11. Omata M, Lesmana LA, Tateishi R, Chen PJ, Lin SM, Yoshida H, et al. Asian Pacific Association for the Study of the Liver consensus recommendations on hepatocellular carcinoma. Hepatol Int. 2010;4(2):43974. doi: 10.1007/s12072-010-9165-7. [PubMed: 20827404].

12. Forner A, Reig ME, de Lope CR, Bruix J. Current strategy for staging and treatment: the BCLC update and future prospects. Semin Liver Dis. 2010;30(1):61-74. doi: 10.1055/s-0030-1247133. [PubMed: 20175034].

13. Xu LF, Sun HL, Chen YT, Ni JY, Chen D, Luo JH, et al. Large primary hepatocellular carcinoma: transarterial chemoembolization monotherapy versus combined transarterial chemoembolizationpercutaneous microwave coagulation therapy. J Gastroenterol Hepatol. 2013;28(3):456-63. doi:10.1111/jgh.12088. [PubMed: 23216261].

14. Ni JY, Liu SS, Xu LF, Sun HL, Chen YT. Transarterial chemoembolization combined with percutaneous radiofrequency ablation versus TACE and PRFA monotherapy in the treatment for hepatocellular carcinoma: a meta-analysis.JCancerRes Clin Oncol. 2013;139(4):653-9. doi: 10.1007/s00432-012-1369-x. [PubMed: 23292073].

15. Wang Y, Deng T, Zeng L, Chen W. Efficacy and safety of radiofrequency ablation and transcatheter arterial chemoembolization for treatment of hepatocellular carcinoma: A meta-analysis. Hepatol Res. 2016;46(1):58-71. doi: 10.1111/hepr.12568. [PubMed: 26265000].

16. Kim JW, Kim JH, Won HJ, Shin YM, Yoon HK, Sung KB, et al. Hepatocellular carcinomas $2-3 \mathrm{~cm}$ in diameter: transarterial chemoembolization plus radiofrequency ablation vs. radiofrequency ablation alone. Eur J Radiol. 2012;81(3):189-93. doi:10.1016/j.ejrad.2011.01.122. [PubMed: 21353417].

17. Komorizono Y, Oketani M, Sako K, Yamasaki N, Shibatou T, Maeda $\mathrm{M}$, et al. Risk factors for local recurrence of small hepatocellular carcinoma tumors after a single session, single application of percutaneous radiofrequency ablation. Cancer. 2003;97(5):1253-62. doi: 10.1002/cncr.11168. [PubMed: 12599233].

18. Buscarini E, Savoia A, Brambilla G, Menozzi F, Reduzzi L, Strobel D, et al. Radiofrequency thermal ablation of liver tumors. Eur Radiol. 2005;15(5):884-94. doi: 10.1007/s00330-005-2652-x. [PubMed: 15754165].

19. Morimoto M, Numata K, Kondou M, Nozaki A, Morita S, Tanaka K. Midterm outcomes in patients with intermediate-sized hepatocellular carcinoma: a randomized controlled trial for determining the efficacy of radiofrequency ablation combined with transcatheter arterial chemoembolization. Cancer. 2010;116(23):5452-60. doi: 10.1002/cncr.25314. [PubMed: 20672352].

20. Takaki H, Yamakado K, Nakatsuka A, Fuke H, Murata K, Shiraki K, et al. Radiofrequency ablation combined with chemoembolization for the treatment of hepatocellular carcinomas $5 \mathrm{~cm}$ or smaller: risk factors for local tumor progression.JVasc Interv Radiol. 2007;18(7):856-61. doi: 10.1016/j.jvir.2007.04.022. [PubMed: 17609444].

21. Morimoto M, Sugimori K, Shirato K, Kokawa A, Tomita N, Saito T, et al. Treatment of hepatocellular carcinoma with radiofrequency ablation: radiologic-histologic correlation during follow-up periods. Hepatology. 2002;35(6):1467-75. doi: 10.1053/jhep.2002.33635. [PubMed: 12029632].

22. Shibata T, Isoda H, Hirokawa Y, Arizono S, Shimada K, Togashi K. Small hepatocellular carcinoma: is radiofrequency ablation combined with transcatheter arterial chemoembolization more effective than radiofrequency ablation alone for treatment?. Radiol- 
ogy. 2009;252(3):905-13. doi: 10.1148/radiol.2523081676. [PubMed: 19567647].

23. Georgiades CS, Hong K, Geschwind JF. Radiofrequency ablation and chemoembolization for hepatocellular carcinoma. Cancer J. 2008;14(2):117-22. doi: 10.1097/PPO.ob013e31816aofac. [PubMed: 18391617].

24. Zhu AX, Abou-Alfa GK. Expanding the treatment options for hepatocellular carcinoma: combining transarterial chemoemboliza- tion with radiofrequency ablation. JAMA. 2008;299(14):1716-8. doi: 10.1001/jama.299.14.1716. [PubMed: 18398085].

25. Takaki H, Yamakado K, Uraki J, Nakatsuka A, Fuke H, Yamamoto N, et al. Radiofrequency ablation combined with chemoembolization for the treatment of hepatocellular carcinomas larger than $5 \mathrm{~cm}$.JVasc Interv Radiol. 2009;20(2):217-24. doi:10.1016/j.jvir.2008.10.019. [PubMed: 19097810]. 